nerungen bei der Linderung depressiver Symptome beziehungsweise der Abschwächung depressionsäquivalenter Verhaltensweisen hilfreich sein kann. Auf der klinischen Ebene setzt die Intervention bei der bekannten kognitiven Problematik depressiver Patienten an, nämlich der (automatischen $=$ nicht bewussten) Verstärkung negativer und Abschwächung positiver Reize. Dieser Mechanismus hat jedoch eine neu- robiologische Grundlage, die mit der Amygdalafunktion verbunden ist. Wenn es auch etwas mechanistisch wirkt, so liegt es doch nahe, mit Biofeedback die entsprechende Amygdalafunktion mithilfe von positiv erlebten Reizen zu trainieren. Dies ist den Autoren nicht nur technisch gut gelungen, sondern hat auch einen erstaunlich ausgeprägten Effekt auf die Depressionssymptomatik. Die Studie ist deshalb wegweisend für die Fortentwicklung einer neurobiologisch informierten Psychotherapie.

Prof. Dr. med. Volker Arolt

Young KD et al. Randomized clinical trial of realtime fMRI amygdala neurofeedback for major depressive disorder: effects on symptoms and autobiographical memory recall. Am J Psychiatry 2017 , online 14. 4.; doi: 10.1176/appi. ajp.2017.16060637

\title{
Antidepressive Psychotherapie: neuronale Konnektivität als Outcome-Prädiktor
}

\section{Wird der Depressionspatient von der geplanten Psychotherapie profitieren? Das lässt sich möglicherweise mit einer bildgebungsbasierten Endophäno- typisierung klären. In einer Experimentalstudie korrelierte die funktionale Konnektivität mit der Therapieresponse.}

U rsache für eine Major Depression (MDD) ist unter anderem eine im Vergleich zu Gesunden veränderte Erregbarkeit bestimmter Hirnstrukturen sowie deren kortikale Vernetzung. So wird die Anhedonie, ein Kernsymptom der MDD, auf eine verringerte neuronale Ansprechbarkeit frontostriataler Regionen auf Belohnungsreize zusammen mit einer herabgesetzten Gewinnantizipation zurückgeführt. In der funktionellen Magnetresonanztomografie (fMRT) ergaben sich bei Untersuchungen mit belohungssystemaktivierenden Tests einerseits krankheitsspezifische Muster als auch interindividuelle Unterschiede bei den einzelnen MDD-Patienten. Diese Zusammenhänge sind nicht nur von akademischem Interesse. Vielmehr könnten sie in der Praxis beispielsweise zur Subgruppenidentifikation von solchen MDD-Patienten herangezogen werden, die von depressionsspezifischen belohnungsreizorientierten Psychotherapien besonders profitieren.

So interessierte sich eine US-amerikanische Forschergruppe für die Frage, in wieweit sich aus den Daten zur funktionellen Konnektivität in frontostriatalen Bereichen von MDD-Patienten Vorhersagen für die Response auf eine Verhaltensaktivationstherapie (Behavioral Activation Treatment for Depression, BATD) ableiten lassen. Die BATD ist ein validiertes psychotherapeutisches Verfahren in der Depressionsbehandlung, bei dem die Patienten lernen, angenehmen Tätigkeiten nachzugehen und ihre Vermeidungsstrategien aufzugeben.

Zur Beantwortung dieser Frage absolvierten 33 ambulante Patienten mit einer MDD zusammen mit 20 passenden Kontrollprobanden zwei per fMRT überwachte Durchgänge des „Monetary Incentive Delay Task“ (MID), einem erprobten neuropsychologischen Testverfahren zur Untersuchung der Belohnungssystemaktivierung. Danach wurden die Patienten mit maximal 15 BATD-Sitzungen behandelt. Die Analyse der Seed-basierten psychophysiologischen Interaktion im fMRT-Scan fokussierte auf die aufgabenspezifische funktionelle Konnektivität während des MID sowie auf eine mögliche Konnektivitätsabschwächung beim zweiten MIDDurchgang.

Bei den MDD-Patienten besserte sich durch die BATD der durchschnittliche Score im Beck Depression Inventory-II um klinisch relevante 10,54 Punkte. In den fMRT-Untersuchungen ergaben sich darüber hinaus während der $\mathrm{Ge}$ winnantizipationsphase des MID deutliche Unterschiede in der funktionellen Konnektivität zahlreicher frontostriataler Seed-Regionen zwischen den MDD-Patienten und den Kontrollper- sonen. Auch innerhalb der Patientengruppe zeigten sich Unterschiede: Eine höhere Konnektivität zwischen dem linken Putamen und dem paracingulären Gyrus in der Antizipationsphase war mit einer verbesserten Response auf die aktivierende Psychotherapie verbunden, so das Ergebnis der hierarchischen linearen Modellierung. Zusätzlich besserten sich die Depressionssymptome vor allem bei Patienten mit einer stärkeren Konnektivitätsabschwächung zwischen einigen frontostriatalen Seeds, kortikalen Bereichen unterhalb des Corpus callosum sowie dem linken paracingulären Gyrus.

\section{Kommentar}

Diese wichtige Studie zeigt in besonderer Weise, wie die wesentlichen Wirkfaktoren einer bestimmten Psychotherapieform (hier der BATD) mit der funktionalen Konnektivität bestimmter Regionen eines neuronalen Netzwerks verbunden sind, und lässt damit umgekehrt Rückschlüsse darauf zu, ob das entsprechende Therapieangebot wirksam wird oder nicht. Die hier vorgestellte Therapieform eignet sich aufgrund ihrer starken Fokussierung auf das Belohnungssystem besonders für die Analyse zu Verbindungen des darunter liegenden neuronalen Netzwerks; die Bedeutung dieser Studie geht jedoch weit über den Einzelfall/die konkret verwendete Therapieform hinaus und weist den Weg für die Translation neurobiologischer Kenntnisse in die klinisch praktische Anwendung in der Psychotherapie.

Prof. Dr. med. Volker Arolt

Walsh $E$ et al. Attenuation of frontostriatal connectivity during reward processing predicts response to psychotherapy in major depressive disorder. Neuropsychopharmacology 2017; 42: $831-43$ 